\title{
El Trabajo Social en el área educativa - Sección Becas Perspectivas futuras en torno al bienestar estudiantil universitario
}

\section{Social Work in the educational area - Section Scholarships Future perspectives regarding student welfare academic}

\author{
Elizabeth Teresa Flores Lazo ${ }^{1 *}$, Juan Andrés Carpio Arévalo ${ }^{1}$ y Jenny Irlanda Tapia Segarra ${ }^{1}$ \\ ${ }^{1}$ Universidad Católica de Cuenca \\ *eflores1@ucacue.edu.ec
}

DOI: https://doi.org/10.26871/killkana_social.v2i4.250

\begin{abstract}
Resumen
Los estudios e investigaciones de vulnerabilidad que busquen dar solución a las problemáticas de los grupos más necesitados sin duda representan un desafío que día a día es el que hacer de la profesión de Trabajo social. El otorgamiento de becas por parte de la Universidad Católica de Cuenca es el reflejo de sus principios primordialmente el servicio al pueblo. La presente investigación tuvo como objetivo principal "Brindar una atención técnica y especializada a los estudiantes postulantes dentro del Departamento de Becas de la Universidad Católica de Cuenca, mediante la creación de un SESE - Sistema de Estratificación Social y Económica, a fin de brindar atención prioritaria a los grupos que la ley señale; y, reducir el margen de error presente en procesos anteriores". Se trabajó con el universo de la población postulante comprendida por 11.545 solicitantes, quienes mediante una encuesta (ficha), llenada a través del Sistema ERP, brindan información de tipo confidencial, fueron consideradas también las observaciones realizadas al momento de las entrevistas personales. Con la aplicación del SESE se evidenció la concordancia de las ponderaciones (puntuaciones) que el sistema otorga a los postulantes; versus, los análisis de preselección realizados de forma manual con la ayuda del programa Excel y personal mediante entrevistas y visitas domiciliarias utilizados anteriormente. Se concluye dejando por sentado la viabilidad y efectividad del SESE, ya que el sistema ha conseguido también distinguir entre los estudiantes más necesitados y en condiciones de vulnerabilidad, doble grado de vulnerabilidad o mayor, de acuerdo a sus distintos indicadores.
\end{abstract}

Palabras clave: Atención prioritaria, Beca, Bienestar, Inclusión, Pobreza, Trabajo social, Vulnerabilidad.

\begin{abstract}
Vulnerability studies and researches that seek to solve the problems of the most needy groups undoubtedly represent a challenge that every day is what to do of the profession of Social Work. The granting of scholarships by the Catholic University of Cuenca is the reflection of its principles primarily service to the people. The main objective of the present research was "To provide technical and specialized attention to prospective students within the Scholarship Department of the Catholic University of Cuenca, through the creation of a SESE - Social and Economic Stratification System, in order to provide priority attention to the groups that the law indicates; and, reduce the margin of error present in previous processes ". We worked with the universe of the applicant population comprised of 11,545 applicants, who through a survey (file), filled through the ERP System, provide confidential information, were also considered the observations made at the time of personal interviews. With the application of the SESE, the concordance of the weights (scores) that the system grants to the applicants was evidenced; versus, preselection analyzes performed manually with the help of the Excel program and staff through interviews and home visits previously used. It is concluded by assuming the viability and effectiveness of the SESE, since the system has also managed to distinguish between the students most in need and in conditions of vulnerability, double degree of vulnerability or higher, according to their different indicators.
\end{abstract}

Key words: Priority attention, Scholarship, Welfare, Inclusion, Poverty, Social work, Vulnerability.

\section{Introducción}

El trabajo social conocido hoy en día como tal, ha atravesado por diversas etapas que se ven reflejadas directamente en la praxis profesional, dichos cambios vistos desde una visión holística humanista han modificado los preceptos del conocido servicio social o asistencia social; sin duda alguna la visión ha sido ambiciosa y con grandes aspiraciones orientadas al bienestar social a través de un desarrollo biopsicosocial de las personas y sus familias. 
De acuerdo a nuevas investigaciones e incremento de metodologías adicionales a las tradicionales con las que surgió el trabajo social han brindado la posibilidad de abarcar de mejor manera las problemáticas sociales de una forma más técnica y conjunta con otras profesiones, que de igual manera tienen incidencia directa con los grupos vulnerables dificulta o impide alcanzar el Buen Vivir de las personas, como lo son: la psicología, el derecho, la antropología, la sociología, la economía, entre otras.

Dichas metodologías han dado respuesta a la complejidad del mundo actual globalizado que acrecienta no solamente los procesos avanzados de tecnologías y comunicación; que, si bien han aportado grandes ventajas especialmente al momento de acortar distancias facilitando el comercio y la negociación entre las partes, inclusive desde continentes que resultaba imposible el intercambio comercial debido a la distancia y tiempo que tomaban la resolución de las negociaciones. Conjuntamente con los aportes positivos han venido de la mano aspectos negativos para las relaciones interpersonales que se ven debilitadas y más aún problemas nocivos para los sectores vulnerables de la población.

El Ecuador mantiene una historia tempestuosa con sus gobiernos; la memoria de escritores reconocidos evidencia la desprotección y olvido que han tenido los grupos más necesitados haciendo caso omiso a sus peticiones y necesidades (Galiano, 1971). Durante la última década el país ha atravesado por una política pública centralizada en el ejecutivo con una presentación izquierdista más conocida como "Socialismo del siglo XXI"; que, si bien ha prestado atención a la población menos beneficiada dando soluciones eventuales y parciales, sin embargo, no ha alcanzado a vislumbrar la problemática desde sus raíces.

Para el año 2017 el Ministerio de Relaciones Laborales Mediante Acuerdo Ministerial N MDT-2016 0300, fija la canasta básica para una familia ecuatoriana según el Instituto Nacional de Estadísticas y Censos se fijó en $\$ 701,93$ dólares y el salario básico unificado para este mismo año comprende los \$375.00 dólares, siendo que el estado asume que dentro de un hogar por lo menos dos personas laboran con este salario básico se tendría como ingreso promedio familiar el valor de $\$ 750.00$ cubriendo en su totalidad los gastos de la canasta básica, inclusive con un excedente de $\$ 48,07$; pues bien, aquella suposición al parecer no resulta siendo real ni aplicable para gran parte de la población Ecuatoriana así como refugiados que han sido acogidos por el estado a razón de situaciones de vulnerabilidad por las que han atravesado (Ministerio de Relaciones Laborales, 2016).

Ecuador cuenta con servicios gratuitos tales como la salud a través de los hospitales regionales del Ministerio de Salud Pública - MSP y el Instituto Ecuatoriano de Seguridad Social - IESS; la educación primaria, secundaria y superior que a través del Ministerio de Educación han logrado eliminar el analfabetismo según informes del Instituto Nacional de Estadísticas y Censos - INEC, ade- más la existencia de otras Instituciones que mediante sus planes, programas y proyectos han mitigado las problemáticas sociales dentro del país trabajando conjuntamente como el Ministerio del Deporte, Ministerio de Inclusión Económica y Social - IESS, quienes velan por el bienestar de la población brindando acceso especial y gratuito a los grupos de atención prioritaria y a los sectores de la sociedad tradicionalmente excluidos como los pueblos indígenas de país. Pues bien, las necesidades de la población continúan latentes.

De las necedades básicas insatisfechas de las familias al parecer yacen una multiplicidad de problemas de las familias ecuatorianas, siendo una de las principales "la Pobreza", que siempre o casi siempre viene fuertemente vinculadas con otras problemáticas por su misma naturaleza de precariedad de servicios, limitación de acceso a una atención de calidad, marginalidad, alimentación precaria, hacinamiento, problemas de salud, salarios bajos, desempleo, abandono o ausentismo escolar, vandalismo, problemas de alcoholismo y drogadicción, embarazos no deseados, abortos clandestinos, matrimonios en adolescentes, maltrato intrafamiliar, entre otros.

Siendo la educación un pilar fundamental para generar conciencia y pensamiento crítico, es considerada una necesidad fundamental que posibilita nuevas formas de vida tanto en el aspecto económico como social, puesto que el proceso de educación es la única herramienta que nivela la igualdad entre las personas.

Dentro del departamento de becas de la Universidad Católica de Cuenca El trabajo social acogiendo los conceptos predefinidos por los pioneros de la materia pretende ser:

El promotor de los principios de los derechos humanos y la justicia social, por medio de la utilización de teorías sobre el comportamiento humano y los sistemas sociales. Específicamente se interesa en la resolución de problemas sociales, relaciones humanas, el cambio social, y en la autonomía de las personas: todo ello en la interacción con su contexto en el ejercicio de sus derechos en su participación como persona sujeto del desarrollo y en la mejora de la sociedad respecto a la calidad de vida en el plano biopsicosocial, cultural, político, económico y espiritual. (Montoya, Zapata, y Cardona, 2002, p. 124).

En el presente artículo se evidenciará el trabajo realizado en pro del conglomerado estudiantil más necesitado que es atendido desde el área de becas, procurando siempre brindar una atención humanista y principalmente reduciendo al máximo el margen de error que, al tratarse de procesos masivos, se evidencia constantemente.

La Universidad Católica de Cuenca, Institución creada desde el 07 de septiembre del 1970, como ideal del Señor Rector Fundador Sacerdote Dr. Cesar Cordero Moscoso. Entre sus principios y valores más arraigados en la comunidad educativa se destacan: Humanismo cristiano; Orientación al servicio a la comunidad; Solidaridad, para 
el acceso a la educación de los más necesitados; Respeto a los Derechos Humanos; Compromiso con la institución; y, Respeto a las tradiciones culturales y religiosas.

Según el Estatuto Orgánico (Universidad Católica de Cuenca, 1970): resulta imprescindible resaltar los valores de la Comunidad Educativa Católica puesto que gracias a ellos el accionar de cada dependencia tiene un camino claro a seguir, resaltando entre ellos:

- Libertad;

- Amor;

- Caridad;

- Paz;

- Verdad;

- Justicia;

- Fraternidad;

- Dignidad;

- Honestidad;

- Solidaridad;

- Respeto a la vida y al medio ambiente;

- Responsabilidad;

- Equidad social y de género;

- $\quad$ Ser útil.

El trabajo social en el área educativa abarca diversas secciones o campos de acción; sin embargo, en el presente trabajo se ha delimitado propiamente a la sección de "becas" por cuanto desde esta actividad se realiza un levantamiento de información sumamente valioso realizado semestralmente, que a su vez brinda especificidad sobre problemáticas que afectan al alumnado más necesitado propiciando ideas de cambios a través de proyectos con la información actualizada constantemente.

Frente a la necesidad de designar y distribuir becas con partida presupuestaria estatal se crea el departamento de Bienestar estudiantil con una sección y jefatura independiente de Becas conformado con profesionales en trabajo social y derecho, quienes ante el mandato constitucional sobre los deberes primordiales del estado, en lo referente a la educación, se encuentra consagrado en el Título II, Capítulo Segundo, Sección quinta, artículos 26, 27, 28 y 29 de la Carta Magna ecuatoriana y, en consonancia aplicativa del inciso final del artículo 356 del mentado instrumento; la Ley Orgánica de Educación Superior - LOES, en armonía a la Disposición Transitoria Decimoctava de la Constitución de la República del Ecuador en su inciso tercero, establece disposiciones específicas para las Universidades Particulares Cofinanciadas -caso de la Universidad Católica de Cuenca-, es decir, para aquellas Universidades que reciben asignaciones del Estado; concernientes a la finalidad de éstos recursos que, según los artículos 30, 74, 77 y 78, en base pragmática de lo que establece el acuerdo 069-2013, emitido por la Secretaría Nacional de Educación, Ciencia, Tecnología e Innovación - SENESCYT, deben distribuirse en asignación de becas a estudiantes en condiciones de vulnerabilidad y/o grupos históricamente excluidos, que por su condición social, económica o discapacidad, les sea difícil iniciar, continuar y/o culminar con éxito su formación profesional. Así:

Las instituciones de educación superior establecerán programas de becas completas o su equivalente en ayudas económicas que apoyen en su escolaridad a por lo menos el $10 \%$ del número de estudiantes regulares; y, serán beneficiarios quienes no cuenten con recursos económicos suficientes, los estudiantes regulares con alto promedio y distinción académica, los deportistas de al to rendimiento que representen al país en eventos internacionales, a condición de que acrediten niveles de rendimiento académico regulados por cada institución y las personas con discapacidad. (Presidencia de la República, 2010)

La Universidad Católica de Cuenca, en mayo del año 2012, crea el Departamento de Becas, dependencia que, evidenciando la necesidad de contar con una metodología eficiente que permita la justa distribución de las becas hacia los estudiantes de bajos recursos económicos y/o que evidencien situaciones sociales desfavorables que les coloquen en niveles de vulnerabilidad permanente o temporal; discapacidad; en reconocimiento a la excelencia académica y el mérito deportivo en primera instancia; y, bajo reglamento interno determina cuatro tipos de becas que responden a estudiantes que presenten uno o algunos de los siguientes componentes de becas:

- Deportistas de alto rendimiento que represente al país en eventos internacionales;

- Excelencia académica;

- Discapacidad; y,

- Situación socioeconómica desfavorable;

Así también existen dos modalidades de becas:

- Beca total

- Beca parcial

El Deportistas de alto rendimiento que represente al país en eventos internacionales recibe un reconocimiento a su aporte hacia la sociedad en general y el incentivo que representa hacia la juventud misma, otorgándosele luego de cumplir con los trámites generales y obligatorios una beca total. La Universidad Católica de Cuenca otorgará este beneficio con el objetivo de estimular el desempeño destacado de las y los estudiantes en la práctica de actividades científicas, deportivas y culturales (Honorable Consejo Universitario de la Universidad Católica de Cuenca, 2013).

La excelencia académica por otro lado permite otorgar beca total al estudiante que muestre un promedio igual o superior a 97/100. La UCACUE con el objetivo de fomentar la excelencia académica en las y los estudiantes regulares, otorgará becas a quienes muestren alto rendimiento académico (Honorable Consejo Universitario de la Universidad Católica de Cuenca, 2013).

El componente por discapacidad permite otorgar beca al estudiante que presente una discapacidad igual o superior al $30 \%$ debidamente calificado por el órgano estatal correspondiente. 
En el caso de las becas otorgadas bajo el componente de situación socioeconómica desfavorable el departamento de becas ha evidenciado una cantidad considerable de problemas al momento de realizar análisis socioeconómicos puesto que la información proporcionada muchas veces responde a errores tanto voluntarios como involuntarios por parte de los postulantes, debido inclusive al desconocimiento de su realidad.

El Trabajo social dentro del departamento de becas ha tenido una ardua tarea como lo es velar por los más necesitados centrando principalmente su atención en las becas por este componente ya que es en este en donde se encuentran un sin número de personas que pese a su situación de vulnerabilidad o inclusive de doble vulnerabilidad han quedado fuera del proceso debido al desconocimiento y errores involuntarios.

En estas circunstancias la premura de realizar un nuevo sistema de estratificación social se ha convertido en una meta a alcanzar en pro del bienestar estudiantil de la Universidad Católica de Cuenca.

\section{Objetivos de la investigación}

\subsection{Objetivo general}

Brindar una atención técnica y especializada a los estudiantes postulantes dentro del Departamento de Becas de la Universidad Católica de Cuenca, mediante la creación de un SESE - Sistema de Estratificación Social y Económica, a fin de brindar atención prioritaria a los grupos que la ley señale; $y$, reducir el margen de error presente en procesos anteriores.

\subsection{Objetivos específicos}

- Sistematizar y clasificar la información recopilada de instituciones públicas y privadas tanto a nivel nacional como internacional relacionadas con la estratificación social y/o económica.

- Crear el SESE (Sistema de Estratificación Social y Económica) del Departamento de Becas de La Universidad Católica de Cuenca.

- Aplicar el SESE dentro del Departamento de Becas de La Universidad Católica de Cuenca. Durante el periodo académico marzo 2017 - agosto 2017

\section{Metodología}

En el presente trabajo investigativo se ha empleado el método cualicuantitativo o método mixto ya que la conjugación de ambos métodos según, Hernández Sampieri "Implican la recolección y el análisis de datos cuantitativos y cualitativos, así como su integración y discusión conjunta, para realizar inferencias producto de toda la información recabada y alcanzar un mayor entendimiento del fenómeno bajo estudio" (Hernández Sampieri, Fernández Collado, y Baptista Lucio, 2010, p. 546).

En este sentido la investigación se fundamenta en datos cualitativos de fuentes propias de la Institución con información recolectada en procesos anteriores, e información externa; así como información cualitativa ya que dentro del departamento se manejan entrevistas personales a cada postulante, visitas domiciliarias, observaciones directas e indirectas que luego de ser contrastadas con información pública otorgada por los mismos estudiantes son cuantificadas.

El paradigma de esta investigación ha sido el críticopropositivo como alternativa para la investigación social, ya que ha permitido interpretar, comprender y explicar el fenómeno de la pobreza y vulnerabilidad en busca de alternativas de solución a las problemáticas sociales presentes en los estudiantes de la Universidad Católica de Cuenca.

\section{Marco Teórico}

\subsection{El Trabajo Social en el Área Educativa sección Becas}

El SESE fue creado con el ideal de agrupar a los postulantes de acuerdo a indicadores que faciliten el análisis de la información proporcionada por los mismos, ha sido una meta para el departamento, ya que luego de constantes diálogos, debates, análisis de información pública y privada, tanto nacional como internacional, ha permitido crear una modalidad de estudios y análisis de casos, que permitirá categorizar al postulante desde dos niveles:

- Grado de vulnerabilidad

- Nivel de situación económica desfavorable o estratificación social

La recolección de información se ha realizado de manera tanto cualitativa como cualitativa, ya sea mediante información consolidada de modelos manejados en otras instituciones y también en base a entrevistas, diálogos con conocedores de la temática ya sea de instituciones públicas y privadas a nivel nacional e internacional.

La ardua labor del Trabajador/a Social en el Área de Becas es de tipo imprescindible su afán de velar por los más necesitados y velar por los derechos de los estudiantes no puede ser sustituida por ninguna otra profesión, sin embargo también se evidencia la necesidad de mantener un equipo multidisciplinario conformado por el área legal y psicológica.

\subsection{Grado de vulnerabilidad}

El grado de vulnerabilidad abarca todas las condiciones especiales que coloquen a las personas o grupos familiares en situaciones de inequidad socioeconómica respecto a otras, tales grados de vulnerabilidad luego de haber sido ampliamente debatidos han sido delimitados a los siguientes: 
Tabla 1 Nivel y Condición de vulnerabilidad

\begin{tabular}{|c|l|}
\hline Nivel & Condición de vulnerabilidad \\
\hline$I$ & $\begin{array}{l}\text { LGBTI - población de lesbianas, gays, bisexuales, travestis, tran- } \\
\text { sexuales e intersexuales tradicionalmente excluidos y discrimina- } \\
\text { dos }\end{array}$ \\
\hline$I$ & Población indígena tradicionalmente excluida \\
\hline$I$ & $\begin{array}{l}\text { Condición de refugiados legalmente certificada por la institución } \\
\text { responsable }\end{array}$ \\
\hline$I$ & Víctimas de desastres naturales \\
\hline 1 & Movilidad estudiantil a razón de educación \\
\hline 1 & Personas con discapacidades dentro del grupo familiar \\
\hline 1 & $\begin{array}{l}\text { Haber estado en condición de PPL (personas privadas de la liber- } \\
\text { tad) durante los últimos cinco años }\end{array}$ \\
\hline$I$ & Enfermedades catastróficas dentro del grupo familiar \\
\hline$I$ & $\begin{array}{l}\text { Beneficiarios del bono de desarrollo humano o cualquier otro bono } \\
\text { de ayuda social estatal dentro del grupo familiar }\end{array}$ \\
\hline$I$ & Condición de pobreza o extrema pobreza \\
\hline$I$ & Mujer en estado de gestación \\
\hline$I 1$ & Total \\
\hline
\end{tabular}

Fuente: Compilado de Documentos Nacionales e Internacionales Elaborado por: Flores, E; Carpio, A; Tapia, J (2017)

Los presentes indicadores de vulnerabilidad son un reflejo de la experiencia que día a día ha sido asentida dentro del departamento de becas especialmente en la fase de entrevistas y visitas domiciliarias que brindan mayor veracidad al momento de manifestar y emitir la recomendación favorable para una beca estudiantil. Cada uno de los indicadores antes mencionados reflejan niveles de vulnerabilidad en algunos casos históricos, como lo son por ejemplo los grupos LGBTI y las personas indígenas, ambos grupos tradicionalmente discriminados y excluidos respectivamente.

La experiencia dentro del departamento ha llevado a concluir en que uno o varios niveles de vulnerabilidad presentes dentro de un grupo familiar evidencia la insatisfacción de las necesidades básicas fundamentales; por tanto, "los Pseudo satisfactores o los satisfactores violadores son los comúnmente utilizados para subsistir; y que en el mejor de los casos mantienen el nivel de vulnerabilidad sin permitir a las personas prosperar y acceder a un mejor estilo de vida de sí mismas y de sus familias" (Max Neef, Elizalde, y Hopenhayn, 1986, p. 43-44).

A manera de ejemplo, un estudiante travesti que se encuentre en condición de refugiado y haya sido víctima del desastre natural recientemente acontecido y con necesidad de movilizarse y cambiar su residencia por estudios; y que, producto del desastre natural tenga una discapacidad y sea beneficiario del bono de persona con discapacidad demostrando con ello una condición de pobreza al ubicarse en el quintil de pobreza número uno; y que además se encuentre en estado de gestación y haya sido una persona privada de la libertad con sentencia ejecutoriada durante los últimos cinco años. Inminentemente esta persona se encontraría en condiciones completamente desfavorables que la harían más vulnerable a ser víctimas de muchos más problemas que dificulten la culminación de sus estudios. Pues bien, el ejemplo no está siendo imaginado y/o lejano a la realidad de muchos estudiantes, pero no necesariamente una persona tendría que presentar todos los niveles de vulnerabilidad para encontrarse más cercano a estar en situaciones de desventaja respecto a otras; ya que, con uno, dos o más niveles de vulnerabilidad presentes indicarían la necesidad de una atención prioritaria.

Las fases del proceso de becas son de suma importancia ya que en base a la información proporcionada en primera instancia por los estudiantes facilitan los indicadores y con ello los niveles de vulnerabilidad presentes. Estas fases son:

- Notificaciones sobre la apertura del sistema de becas en los medios de comunicación universitaria

- Postulación estudiantil mediante una ficha socioeconómica a ser llenada

- Análisis y preselección de los perfiles idóneos para ser becados

- Entrevistas y corroboración de la información proporcionada en la ficha socioeconómica

- Visitas domiciliarias

- Elaboración de informes sociales

- Aprobación o negación por parte del consejo de becas

La postulación estudiantil con información clara y veraz es imprescindible para facilitar el análisis y evitar percances al momento de la entrevista, en donde comúnmente se determinan inconsistencias en la información, pues en contraste con la documentación solicitada la información declarada en la ficha socioeconómica no refleja la realidad del estudiante y su grupo familiar.

Las personas LGBTI han sido consideradas dentro de los 11 niveles de vulnerabilidad ya que se ha evidenciado que la sociedad ecuatoriana muestra fuerte discriminación por estos grupos de personas dificultando la posibilidad de acceder aún empleo digno llevándoles en algunos casos hacia la prostitución.

Las personas indígenas, han sido grupos por quienes el estado ha tomado conciencia sobre la desprotección histórica que ha tenido hacia estos sectores creando la figura propiamente de "pueblos indígenas tradicionalmente excluidos" para quienes se pretende generar inclusión social mediante la atención prioritaria, hoy por hoy muchos jóvenes y adultos de los pueblos indígenas se encuentran educándose y en su gran mayoría serán una de las primeras generaciones de sus hogares en tener educación de tercer nivel. Los grupos indígenas dedicados principalmente a actividades de agricultura y ganadería relejan condiciones de pobreza y desprotección estatal ante la escasez de las obras públicas y la comercialización injusta de sus productos.

Las personas que se encuentran legalmente en condición de refugiado, responde a una responsabilidad estatal de acoger a un extranjero o extranjera como ciudadano mismo del estado ecuatoriano y brindarle toda la protección y seguridad posible, ya que dicha condición refleja situaciones de vulnerabilidad y doble vulnerabilidad dentro de su territorio natal, viéndose obligadas a huir o abandonar su país por su seguridad y vida misma. Según la ACNUR las personas refugiadas son: 
Todas las personas que huyen de su país a causa de un temor fundado de persecución por motivos de raza, religión, nacionalidad, opinión política o pertenencia a un grupo social y que, no pueden o, a causa de dichos temores, no quieren acogerse a la protección de su país; o que careciendo de nacionalidad y hallándose a consecuencia de tales acontecimientos fuera del país de residencia habitual, no pueden o no quieren regresar a él. También son personas refugiadas quienes huyen de su país porque su vida, seguridad o libertad han sido amenazadas por la violencia generalizada, la agresión extranjera, los conflictos internos, la violación masiva de los derechos humanos u otras circunstancias que hayan perturbado gravemente el orden público. (Alto Comisionado de las Naciones Unidas para los Refugiados - ACNUR, 2010)

Las personas y/o grupos familiares víctimas de desastres naturales tales como: terremotos, huracanes, incendios, erupciones volcánicas, inundaciones, maremoto, entre otros. Muestran una o varias condiciones de vulnerabilidad puesto que en estos acontecimientos inesperados las pérdidas humanas y materiales dificultan la satisfacción de las necesidades humanas básicas durante un tiempo considerable.

La movilidad estudiantil a razón de educación refleja un abandono total o parcial dependiendo la distancia del hogar del estudiante, lo cual representa un egreso económico alto y adicional, ya que comúnmente el ingreso total familiar se debe dividir para poder mantener prácticamente dos hogares al mismo tiempo.

La presencia de personas con discapacidad y/o enfermedades catastróficas dentro de un grupo familiar representa un egreso constante en la familia, además si se tratare de enfermedades que imposibiliten a una persona o cuando se tratare de discapacidades porcentualmente altas se presume la dificultad de que una o varias personas en posibilidad de laborar no pueda hacerlo por cuidado de las mismas. La Ley de Discapacidades del Ecuador en su Art. 6 menciona al respecto de la Persona con discapacidad. "Se considera persona con discapacidad a toda aquella que, como consecuencia de una o más deficiencias físicas, mentales, intelectuales o sensoriales, con dependencia de la causa que la hubiera originado, ve restringida permanentemente su capacidad biológica, sicológica y asociativa para ejercer una o más actividades esenciales de la vida diaria" (Presidencia de la República, 2012).

Las personas que se han encontrado en condición de PPL (persona privada de la libertad) durante los últimos 5 años; evidencian comúnmente situaciones familiares de disfuncionalidad en su dinámica; a más de ello, la dificultad que representa para estas personas acceder a algún trabajo como consecuencia de los antecedentes legales vuelven más vulnerable a estas personas a verse involucrados en trabajos ilegales y/o injustos en condiciones inequitativas respecto a otras. El compromiso social de inclusión siempre presente en la UCACUE busca lograr una reinserción social de estas personas de una manera óptima para sí mismos y sus familias.

Las personas beneficiarias del bono de desarrollo humano o cualquier otro bono y/o ayuda social estatal dentro del grupo familiar denotan en el análisis socioeconómico condiciones directas de pobreza, ya que estas ayudas sociales estatales son direccionadas previo levantamiento de investigación de campo propiamente a los grupos familiares que reflejen pobreza o extrema pobreza.

Las mujeres en estado de gestación se encuentran dentro de los 11 niveles de vulnerabilidad ya que la ley de estado ecuatoriano considera a las mujeres en estado de gestación como grupo de atención prioritaria. A más de ello la dificultad por al cual atraviesan las estudiantes en estado de gestación las coloca en condiciones de desventaja respecto a otras por el ausentismo propio del maternidad y demás problemas económicos.

\subsection{Nivel de situación económica desfavorable o estrati- ficación social}

Luego de considerar a todos los grupos de atención prioritaria con niveles de vulnerabilidad, se ha visto la necesidad de jerarquizar los niveles económicos mediante un sistema de estratificación que determine los niveles de pobreza en el que se encuentren los estudiantes y sus grupos familiares a fin de priorizar recursos y destinar becas hacia los más necesitados.

Según la Encuesta de Estratificación del Nivel Socioeconómico Realizada por el INEC considera que la estratificación social debe utilizar variables que determinan las condiciones de pobreza de acuerdo a distintos indicadores (Instituto Nacional de Estadisticas y Censos, 2011), entre ellos:

- $\quad$ Tipo de vivienda

- Condición de la vivienda

- Espacios con los que cuenta la vivienda

- Número de habitaciones

- Numero de baños

- Acceso a servicios básicos

- Acceso a la educación en sus diferentes niveles y tipos

- Acceso a la salud (pública o privada)

- $\quad$ Seguridad social (pública, privada, ninguna)

- Tipo de trabajo

- Acceso a la seguridad social, seguro privado u otros

- Posesión de bienes inmuebles

- Posesión de bienes vehiculares

- Posesión de electrodomésticos

- Número de integrantes del grupo familiar

- Ingreso total del grupo familiar

La estratificación determina un valor dependiendo del grado de importancia que se considere en cada variable. Dándonos un máximo de 1000 mil puntos y una clasificación de estratificación de 6 categorías o seis niveles de estratificación. 


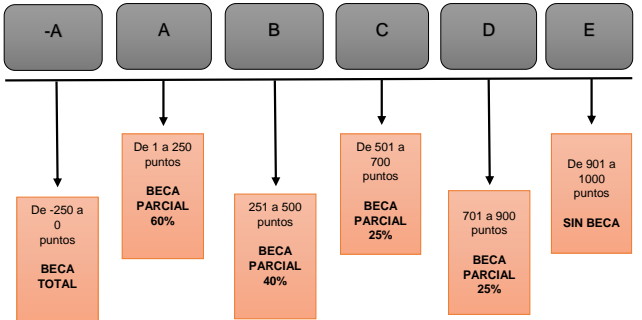

Figura 1. Sistema de Estratificación Social y Económica - SESE

Es importante recalcar que los valores dados en los niveles de vulnerabilidad serán en negativo procurando así brindar especial atención en estos sectores.

\subsection{Bienestar estudiantil universitario}

Del levantamiento de información principalmente de los grupos vulnerables se pretende extender proyectos que velen por el bienestar de los estudiantes de la Universidad Católica de cuenca, sectorizando las necesidades y los recursos de acuerdo al nivel de premura que se considere por el o los factores de riesgo que disminuyan o dificulten la continuidad y culminación de los estudios.

Llevando acabo la estratificación social conjuntamente con los niveles de vulnerabilidad en el proceso de becas, durante el periodo académico marzo 2017 - agosto 2017 en el Departamento de Becas de la Universidad Católica de Cuenca se han obtenido resultados efectivos y prácticos al momento de realizar tanto la preselección, como las demás etapas del proceso. Tales resultados fueron los siguientes.

\section{Análisis e interpretación de resultados}

Tabla 2 Universo de la población estudiantil solicitante y no solicitante

\begin{tabular}{lcc}
\hline Descripción & $\begin{array}{c}\text { Total } \\
\text { Cantidad }\end{array}$ & $\begin{array}{c}\text { Total } \\
\text { Porcentaje }\end{array}$ \\
\hline NO POSTULANTES & 8706 & $76 \%$ \\
POSTULANTES & 2839 & $24 \%$ \\
\hline Total General & $\mathbf{1 1 5 4 5}$ & $\mathbf{1 0 0 \%}$ \\
\hline
\end{tabular}

Fuente: Encuestas de postulantes (Sistema ERP)

Elaborado por: Flores, E; Carpio, A; Tapia, J (2017)

UNIVERSO DE LA POBLACIÓN ESTUDIANTIL (SOLICITANTES Y NO SOLICITANTE)

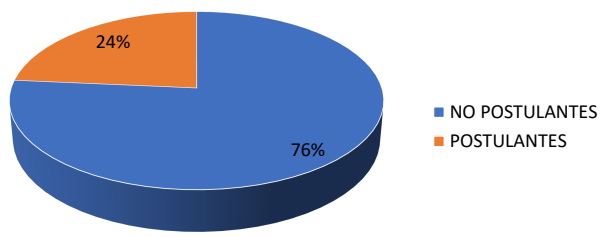

Figura 2. Universo de la población estudiantil solicitante y no solicitante Elaborado por: Flores, E; Carpio, A; Tapia, J (2017)
Análisis e interpretación: Del universo de la población estudiantil de la Universidad Católica de Cuenca ubicada en 11.545 estudiante; 8.706 estudiantes equivalente al $76 \%$ NO postularon al proceso de becas mediante el sistema informático ERP; y, apenas el $24 \%$ correspondiente a 2.839 estudiantes postularon a fin de obtener una beca. Pese a la difusión mediante los medios de comunicación institucionales la cantidad de estudiantes postulantes se refleja reducida considerando el universo de la población estudiantil.

Tabla 3 Encuestas de postulantes (Sistema ERP)

\begin{tabular}{lcc}
\hline Descripción & $\begin{array}{c}\text { Suma de } \\
\text { Cantidad }\end{array}$ & $\begin{array}{c}\text { Suma de } \\
\text { Porcentaje }\end{array}$ \\
\hline $\begin{array}{l}\text { Postulantes preseleccionados que } \\
\text { pasan a entrevista }\end{array}$ & 1589 & $56 \%$ \\
Postulantes no preseleccionados & 1250 & $44 \%$ \\
\hline Total General & $\mathbf{2 8 3 9}$ & $\mathbf{1 0 0} \%$ \\
\hline
\end{tabular}

Fuente: Encuestas de postulantes (Sistema ERP)

Elaborado por: Flores, E; Carpio, A; Tapia, J (2017)

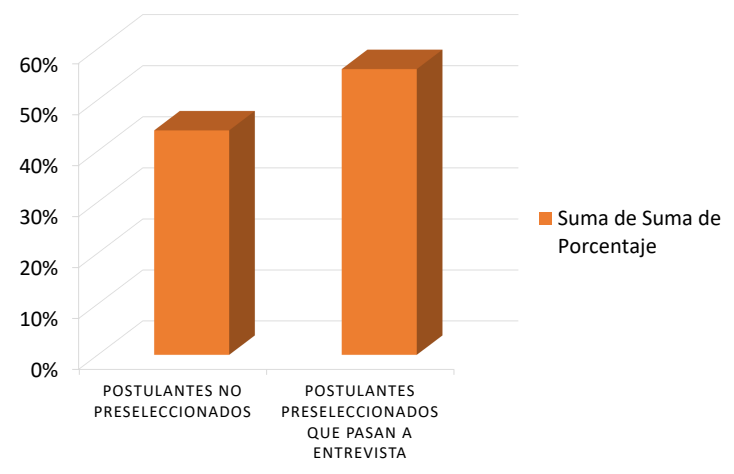

Figura 3. Universos de la población estudiantil solicitante Becados y no becados

Elaborado por: Flores, E; Carpio, A; Tapia, J (2017)

Análisis e interpretación: Del universo de la población estudiantil POSTULANTE en el proceso de becas; 1.589 estudiantes correspondientes al $56 \%$ fueron preseleccionados para acceder a una beca, pasando a la etapa de entrevista. Por otra parte, el $44 \%$ de estudiantes solicitantes correspondiente a 1250 estudiantes no pasan a la etapa de entrevistas por diferentes causales, entre algunos de los más comunes presentes: inconsistencias en la información económica, datos en blanco que imposibilitan el análisis socioeconómico del grupo familiar por parte del equipo de trabajo social y perfil socioeconómico no idóneo para acceder a una beca.

Importante recalcar que esta preselección excluye a los estudiantes que aplican por el componente de excelencia académica ya que en este tipo de componente no incide la condición social y/o económica ejecutándose propiamente como un reconocimiento al mérito académico. 
Tabla 4 Encuestas de postulantes (Sistema ERP)

\begin{tabular}{lcc}
\hline Descripción & $\begin{array}{c}\text { Suma de } \\
\text { Cantidad }\end{array}$ & $\begin{array}{c}\text { Suma de } \\
\text { Porcentaje }\end{array}$ \\
\hline Deportista de alto rendimiento & 13 & $1 \%$ \\
Discapacidad & 79 & $6 \%$ \\
Exelencia académica & 211 & $15 \%$ \\
Recursos económicos insuficientes & 1075 & $78 \%$ \\
\hline Total general & $\mathbf{1 3 7 8}$ & $\mathbf{1 0 0 \%}$ \\
\hline
\end{tabular}

Fuente: Encuestas de postulantes (Sistema ERP)

Elaborado por: Flores, E; Carpio, A; Tapia, J (2017)

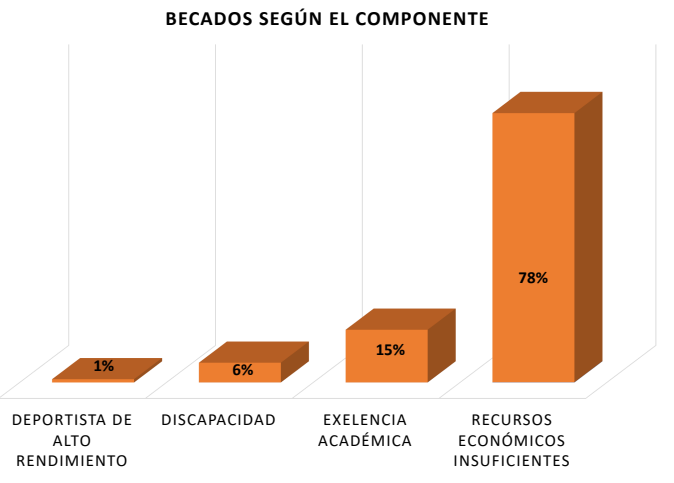

Figura 4. Becados según el componente

Elaborado por: Flores, E; Carpio, A; Tapia, J (2017)

Análisis e Interpretación: De las becas otorgadas según el componente de aplicación los resultados han sido los siguientes: el $1 \%$ correspondiente a 13 estudiantes han obtenido beca total por el componente de Deportista de Alto Rendimiento; el $6 \%$ correspondiente a 79 estudiantes han obtenido beca total por el componente de Discapacidad; el $15 \%$ correspondiente a 211 estudiantes han obtenido beca total por el componente de Excelencia Académica según lo indica el reglamento de becas. Así también el $78 \%$ de estudiantes correspondiente a 1075 estudiantes han obtenido beca por el componente de Recursos económicos insuficientes. Teniendo en la culminación del proceso 1378 estudiantes becados.

Tabla 5 Encuestas de postulantes (Sistema ERP)

\begin{tabular}{lcc}
\hline Descripción & $\begin{array}{c}\text { Suma de } \\
\text { Cantidad }\end{array}$ & $\begin{array}{c}\text { Suma de } \\
\text { Porcentaje }\end{array}$ \\
\hline $\begin{array}{l}\text { Estudiante en estado de gestación } \\
\begin{array}{l}\text { Estudiante PPL (durante los últimos } \\
5 \text { años) }\end{array}\end{array}$ & 2 & $0,25 \%$ \\
$\begin{array}{l}\text { Estudiantes de los pueblos y nacio- } \\
\text { nalidades indígenas }\end{array}$ & 92 & $0,17 \%$ \\
$\begin{array}{l}\text { Estudiantes de identificación LGB- } \\
\text { TI }\end{array}$ & 4 & $7,73 \%$ \\
$\begin{array}{l}\text { Grupos familiares víctimas de } \\
\text { desastres naturales }\end{array}$ & 26 & $0,34 \%$ \\
\hline Total general & $\mathbf{1 1 9 0}$ & $2,18 \%$ \\
\hline
\end{tabular}

Tabla 5 Encuestas de postulantes (Sistema ERP) ... continuación

\begin{tabular}{lcc}
\hline Descripción & $\begin{array}{c}\text { Suma de } \\
\text { Cantidad }\end{array}$ & $\begin{array}{c}\text { Suma de } \\
\text { Porcentaje }\end{array}$ \\
\hline $\begin{array}{l}\text { Integrantes del grupo familiar bene- } \\
\text { ficiarios del BDH }\end{array}$ & 138 & $11,60 \%$ \\
$\begin{array}{l}\text { Integrantes del grupo familiar con } \\
\text { discapacidad }\end{array}$ & 229 & $19,24 \%$ \\
$\begin{array}{l}\text { Integrantes del grupo familiar con } \\
\text { enfermedad catastrófica }\end{array}$ & 155 & $13,03 \%$ \\
$\begin{array}{l}\text { Integrantes del grupo familiar en } \\
\text { condición de refugiados }\end{array}$ & 1 & $0,08 \%$ \\
$\begin{array}{l}\text { Situación de pobreza o pobreza ex- } \\
\text { trema (quintil uno o dos) }\end{array}$ & 540 & $45,38 \%$ \\
\hline Total general & $\mathbf{1 1 9 0}$ & $\mathbf{1 0 0 \%}$ \\
\hline
\end{tabular}

Fuente: Encuestas de postulantes (Sistema ERP)

Elaborado por: Flores, E; Carpio, A; Tapia, J (2017)

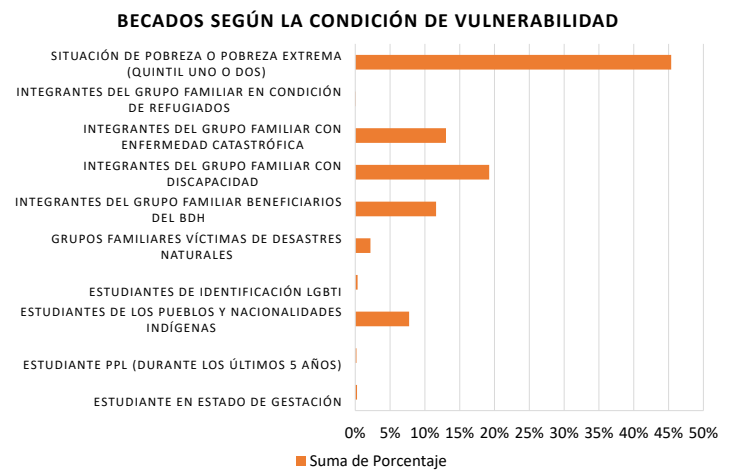

Figura 5. Becados según la condición de vulnerabilidad

Elaborado por: Flores, E; Carpio, A; Tapia, J (2017)

Análisis e interpretación: De las becas otorgadas según el componente de Recursos Económicos insuficientes se han otorgado becas de acuerdo al nivel o niveles de vulnerabilidad y por la situación de pobreza o pobreza extrema, los datos representan el nivel de vulnerabilidad de los estudiantes solicitantes quienes pueden presentar uno o varios niveles; por tanto, la gráfica no representan el total de las becas por condición de vulnerabilidad sino las condiciones de vulnerabilidad que presentan los postulantes de Recursos Económicos Insuficientes en general.

El $0.25 \%$ de solicitantes correspondiente a 3 estudiantes se encuentran en estado de gestación; el $0.17 \%$ de solicitantes correspondiente a 2 estudiantes han sido PPL (personas privadas de la libertad) con sentencia ejecutoriada durante los 5 últimos años; el $7.73 \%$ de solicitantes correspondientes a 92 estudiantes pertenecen a los pueblos y nacionalidades indígenas tradicionalmente discriminados y olvidados; el $0.34 \%$ correspondiente a 4 estudiantes presentan identificación de género de alguno de los grupos LGBTI; EL $2.18 \%$ correspondiente a 26 estudiantes y sus grupos familiares han sido víctimas de desastres naturales; el $11.60 \%$ correspondiente al 138 estudiantes presentan integrantes del grupo familiar beneficiarios del bono de desarrollo humano o cualquier otro programa de ayuda económica estatal; el 19.24\% correspondiente al 229 estudiantes presentan integrantes del grupo familiar 
con discapacidades; el $13.03 \%$ correspondiente a 155 estudiantes presentan integrantes del grupo familiar con enfermedades catastróficas; el $0.08 \%$ correspondiente a 1 estudiante presentan integrantes del grupo familiar o de sí mismo condición de refugiado; el $45.38 \%$ correspondiente a 540 estudiantes reflejan condiciones de pobreza o pobreza extrema.

\section{Conclusiones}

Con el sistema de estratificación SESE implementado las perspectivas futuras de disminuir y/o erradicar el margen de error e inconsistencias en la información emitida por los estudiantes y mantener un sistema técnico matemático que permita clasificar los niveles de pobreza y atender principalmente a los estudiantes más necesitados se han obtenido resultados sumamente alentadores que han acercado por extremo al objetivo de la investigación.

El sistema de estratificación social ha conseguido distinguir entre los estudiantes más necesitados y en condiciones de vulnerabilidad, doble grado de vulnerabilidad o mayor, de acuerdo a sus distintos indicadores.

La atención prioritaria a sectores que la ley indica muestran niveles de condición económica y social baja concomitantemente con el sistema de estratificación social

\section{Recomendaciones}

Realizar constantes seguimientos a los estudiantes que se encuentren en la categoría -A del sistema de estratificación social.

Crear proyectos sociales y/o académicos que permitan brindar tutorías de diferente índole a los estudiantes que presentan doble condición de vulnerabilidad o mayor en coordinación con otros departamentos de la Universidad.

Crear proyectos inclusivos para los estudiantes que muestren condiciones especiales de discriminación o desigualdad respecto a otros.

\section{Referencias Bibliográficas}

Alto Comisionado de las Naciones Unidas para los Refugiados - ACNUR. (2010). Guía informativa - ACNUR. Descargado de http://www.acnur.org/fileadmin/ scripts/doc.php?file=fileadmin/ Documentos/RefugiadosAmericas/ Ecuador/Guia_informativa_sobre _refugio_en_Ecuador

Galiano, E. (1971). Las Venas Abiertas de América Latina. Montevideo: Siglo XXI Editores.
Hernández Sampieri, R., Fernández Collado, C., y Baptista Lucio, M. d. P. (2010). Metodología de la Investigación. México: McGRAWHILL/INTERAMERICANA EDITORES,S.A. DE C.V.

Honorable Consejo Universitario de la Universidad Católica de Cuenca. (2013). Reforma al Reglamento de Becas y Ayudas Económicas de Grado y Postgrado. Cuenca, Azuay, Ecuador: EDUNICA.

Instituto Nacional de Estadisticas y Censos. (2011). Encuesta de Estratificación del Nivel Socioeconómico. Descargado de http: / / www . ecuadorencifras.gob.ec/ documentos/web-inec/Estadisticas _Sociales/Encuesta_Estratificacion _Nivel_Socioeconomico/111220_NSE _Presentacion.pdf

Max Neef, M., Elizalde, A., y Hopenhayn, M. (1986). Desarrollo a Escala Humana una opción para el futuro. Chile: CEPAUR.

Ministerio de Relaciones Laborales. (2016). Ministerio de Relaciones Laborales. Descargado de http://www.trabajo.gob.ec/ wp-content/uploads/downloads/2017/ 01/AM-SBU-MDT-2016-0300.pdf

Montoya, G., Zapata, C., y Cardona, B. (2002). Diccionario especializado de trabajo social. Medellín: Universidad de Antioquia.

Presidencia de la República. (2010). Ley Orgánica de Educación Superior. Quito: Lexis Finder.

Presidencia de la República. (2012). Ley Orgánica de Discapacidades (Inf. Téc.). Quito.

Universidad Católica de Cuenca. (1970). Estatuto Orgánico. Cuenca, Azuay, Ecuador: EDUNICA.

Recibido: 18 de abril de 2018 Aceptado: 4 de diciembre de 2018 
